\section{Hunting down HER2}

\author{
By Lev Osherovich, Senior Writer
}

Two studies suggest new ways to target tumors resistant to Herceptin trastuzumab, an anti-HER2 antibody marketed by Genentech Inc. and Roche to treat breast cancer. A German group has generated HER2seeking natural killer cells, ${ }^{1}$ whereas an Italian team has engineered a strain of herpes simplex virus that hones in on HER2 instead of its natural receptor. ${ }^{2}$ In both cases, the targeted HER2-overexpressing cells were eliminated.

The HER2 (ERBB2) protein is present at low levels in many normal cells, where it modulates the activity of epidermal growth factor receptor (EGFR). But in many breast, ovarian and stomach carcinomas, HER2 expression is amplified, leading to excessive growth-promoting signals.

Herceptin, which posted U.S. sales of $\$ 1$ billion through the first nine months of 2008 , works by binding to the extracellular portion of HER2. This is thought to recruit complement
"The challenges to developing this protocol to an industrial scale are numerous."

-François Romagné, Innate Pharma S.A.
In experiments with NK cells isolated from ovarian cancer patients and transfected with the chimeric HER2-specific receptor, the team observed a positive response to tumor cells isolated from the same patient. Ovarian cancer cells express HER2 but do not generally respond to Herceptin, possibly because of the relatively low levels of HER2 expressed on these cells. ${ }^{5}$

Finally, in a mouse model of ovarian tumor growth, the HER2-seeking NK cells prevented tumor growth and increased survival compared with what was seen using either mock-treated NK cells or no NK cells.

"We showed for the first time that these efficient killer cells, which exhibit many other antitumor characteristics, can be specifically targeted toward HER2-positive tumors," said Anna Kruschinski, a graduate student at the Max-Delbrück Center and lead author of the study.

Kruschinski believes the approach offers advantages over Herceptin.

"Herceptin therapy is currently used to treat only HER2-amplified tumors, representing one-third of HER2-positive tumors," she said. "Our approach succeeded in targeting all HER2-positive tumors, including but not limited to this one-third of patients. Therefore, our approach might replace or be combined with Herceptin."

Mark Sliwkowski, a research oncology staff scientist at Genentech, suggested that any therapies developed from the work published in PNAS would face stiff competition from more straightforward mAbs and small molecules.

"It would be their challenge to show superifactors and innate immune cells such as natural killer (NK) cells to attack the tumor.

It is unclear why certain HER2-positive tumors are resistant to Herceptin, although several mechanisms have been proposed. ${ }^{3}$ Both new approaches avoid this uncertainty, however, by pursuing alternative routes to attacking cells that bear the protein.

\section{The NK of time}

The NK cell study was published in the Proceedings of the National Academy of Sciences by a team led by Jehad Charo, a staff scientist at the Max-Delbrück Center for Molecular Medicine. It is the team's most recent effort to design chimeric receptors consisting of an immune response-triggering intracellular domain and an external tumor-recognizing domain. Such chimeric receptors are then expressed on the surface of immune cells.

As described in an earlier paper, ${ }^{4}$ the team previously developed a HER2-specific chimeric receptor consisting of an intracellular region derived from a $\mathrm{T}$ cell surface protein and an extracellular region derived from a HER2 antibody different from Herceptin.

In the new paper, Charo's team transfected human NK cells with the construct for the chimeric receptor and cultured them in the presence of HER2-positive and HER2-negative cancer cells. The transfected cells became activated in the presence of HER2-positive cancer cells, releasing cytoplasmic granules and interferon- $\gamma$. But HER2-negative cells did not activate the NK cells. ority or equivalency" to marketed drugs such as Herceptin and Tykerb lapatinib, he said.

Tykerb, a small molecule inhibitor of HER2 and EGFR, is marketed by GlaxoSmithKline plc to treat HER2-positive breast cancer and is in Phase II and Phase III trials in other oncology indications.

In addition, Sliwkowski said the HER2-targeted NK cells may work a little too well. He noted that in addition to attacking HER2-overexpressing tumors, the NK cells also recognized a cancer cell line with low levels of HER2. This raises concerns that engineered NK cells could indiscriminately attack healthy and cancer cells alike.

Kruschinski told SciBX that she has not tested the effect of HER2seeking NK cells on healthy epithelial cells.

"I am not surprised that they can get NK cells to attack HER2-positive cancer cells," said R.J. Tesi, CEO of Coronado Biosciences Inc. "What may be novel is the ability of NK cells that are not activated [in advance] to kill cancer cells" as a result of binding the target cells via HER2.

According to Tesi, previous studies have suggested that reintroduced NK cells first must be primed with cytokines or exposure to cancer antigens prior to activation, ${ }^{6}$ a conclusion potentially challenged by Kruschinski and Charo's study.

Coronado's CNDO102, a cell-based therapy that primes NK cells ex vivo to recognize and kill tumor cells, is in preclinical development to treat lymphatic cancers. 
François Romagné, CSO of Innate Pharma S.A., said the PNAS article "stresses that NK cells are important mediators of anticancer effects" and thus is in line with his company's efforts to stimulate NK activity to treat cancer. However, he said, getting sufficient numbers of patient-derived NK cells for this approach to work is a tall order.

"The challenges to developing this protocol to an industrial scale are numerous," Romagné said. "It combines cell therapy and genetic modification of the NK cells."

Innate is developing IPH2101, an NK cell-activating antibody in Phase I trials to treat acute myeloid leukemia (AML) and mul-

\author{
"Our recombinant HSV \\ shares its target with \\ Herceptin." \\ - Gabriella Campadelli-Fiume, \\ University of Bologna
}

"Our recombinant HSV shares its target with Herceptin," Campadelli-Fiume told SciBX. "A major advantage over Herceptin is that the virus replicates, a feature that enables the therapeutic viruses to compete with proliferating cancer cells" to outpace tumor growth.

Campadelli-Fiume believes her HSV-1 variant will be useful for Herceptin-resistant HER2-positive tumors such as ovarian cancer, but it could also be used in combination with Herceptin in breast cancer.

Genentech's Sliwkowski said the strategy was "very clever," but he was concerned that previous attempts at building targeted oncolytic viruses have not yet succeeded.

MediGene AG and BioVex Inc. have HSV1-based cancer therapeutics in Phase II trials for liver cancer and various cancer indications, respectively. Both companies declined to comment on Campadelli-Fiume's study.

Attenuated strains typically contain engineered mutations that diminish the ability of a virus to replicate in normal cells as well as other mutations that make cancer cells more attractive hosts. The problem is that these attenuations also may decrease potency against the actual tumor.

Campadelli-Fiume noted that her team's HSV-1 strain remains fully virulent but simply cannot enter normal cells.

The strain's safety profile "is a consequence of the fact that it can only infect, replicate and destroy HER2-positive cells," said Campadelli-Fiume.

Sliwkowski suggested that Campadelli-Fiume's team should test the engineered virus against a larger panel of HER2-positive cancer cell lines. However, Campadelli-Fiume is already moving into mouse models of cancer.

She told SciBX the University of Bologna has filed patents on the technology and is negotiating licensing agreements with undisclosed companies.

Osherovich, L. SciBX 1(43); doi:10.1038/scibx.2008.1039

Published online Dec. 4, 2008

REFERENCES

1. Kruschinski, A. et al. Proc. Natl. Acad. Sci. USA; published online Nov. 11, 2008; doi:10.1073/pnas.0804788105

Contact: Jehad Charo, Max-Delbrück Center for Molecular Medicine, Berlin, Germany e-mail: j.charo@mdc-berlin.de

2. Menotti, L. et al. J. Virol.; published online Aug. 6, 2008; doi:10.1128/JVI.01133-08

Contact: Gabriella Campadelli-Fiume, University of Bologna, Bologna, Italy

e-mail: gabriella.campadelli@unibo.it redirect the virus exclusively to HER2-expressing cells, the team introduced mutations that eliminated binding to one of the host proteins. They then swapped a Herceptin-derived antibody fragment in place of the $\mathrm{gD}$ region that binds the second host protein.

The resulting engineered virus infected HER2-positive cells such as cultured ovarian cancer cells but could not recognize a variety of human cell lines that lacked HER2.

To prove that the virus relied on HER2 for infection, the team pretreated ovarian cancer cells with Herceptin. This prevented the engineered virus from infecting the cell because its new target was now blocked by the antibody. In contrast, treatment with antibodies against one of the normal host receptors did not block the engineered virus but did prevent infection by wild-type HSV.

\section{Valabrega, G. et al. Ann. Oncol. 18, 977}

Chmielewski, M. et al. J. Immunol. 173, 7647-7653 (2004)

5. Longva, K.E. et al. Int. J. Cancer 116, 359-367 (2005)

6. North, J. et al. J. Immunol. 178, 85-94 (2007)

COMPANIES AND INSTITUTIONS MENTIONED

BioVex Inc., Woburn, Mass.

Coronado Biosciences Inc., San Diego, Calif.

Genentech Inc. (NYSE:DNA), South San Francisco, Calif.

GlaxoSmithKline plc (LSE:GSK; NYSE:GSK), London, U.K.

Innate Pharma S.A. (Euronext:IPH), Marseille, France

Max-Delbrück Center for Molecular Medicine, Berlin, Germany

MediGene AG (Xetra:MDG), Martinsried, Germany

Novo Nordisk A/S (CSE:NVO; NYSE:NVO), Bagsvaerd, Denmark

Roche (SWX:ROG), Basel, Switzerland

University of Bologna, Bologna, Italy 